\title{
DIGITALCOMMONS
}

@WAYNESTATE-

Wayne State University

Mathematics Faculty Research Publications

Mathematics

$10-1-2005$

\section{Remarks on Risk-Sensitive Control Problems}

José Luis Menaldi

Wayne State University, menaldi@wayne.edu

Maurice Robin

Ecole Polytechnique

\section{Recommended Citation}

Menaldi, JL. \& Robin, M. Appl Math Optim (2005) 52: 297. doi: 10.1007/s00245-005-0829-y

Available at: https://digitalcommons.wayne.edu/mathfrp/55

This Article is brought to you for free and open access by the Mathematics at DigitalCommons@WayneState. It has been accepted for inclusion in Mathematics Faculty Research Publications by an authorized administrator of DigitalCommons@WayneState. 


\title{
Remarks on Risk-sensitive Control Problems
}

\author{
JosÉ-LUIS MENALDI \\ Wayne State University \\ Department of Mathematics \\ Detroit, Michigan 48202, USA \\ (e-mail: jlm@math.wayne.edu)
}

\author{
MAURICE Robin \\ Ecole Polytechnique \\ 91128 Palaiseau, France \\ (e-mail: maurice.robin@polytechnique.fr)
}

\begin{abstract}
The main purpose of this paper is to investigate the asymptotic behavior of the discounted risk-sensitive control problem for periodic diffusion processes when the discount factor $\alpha$ goes to zero. If $u_{\alpha}(\theta, x)$ denotes the optimal cost function, $\theta$ being the risk factor, then it is shown that $\lim _{\alpha \rightarrow 0} \alpha u_{\alpha}(\theta, x)=\xi(\theta)$ where $\xi(\theta)$ is the average on ]0, $\theta[$ of the optimal cost of the (usual) infinite horizon risk-sensitive control problem.
\end{abstract}

\section{Introduction}

Let us consider a simple stochastic control model given by the following Itô equation

$$
\mathrm{d} x_{t}=b\left(x_{t}, v_{t}\right) \mathrm{d} t+\sqrt{2} \mathrm{~d} B_{t}, \quad x_{0}=x,
$$

where $x$ is the state of the system in $\mathbb{R}^{d}$ and $v$ is the control in $\mathbb{R}^{m}$. For a parameter $\theta \neq 0$, the functional cost is

$$
I_{\alpha}(\theta, x, v)=\frac{1}{\theta} \ln \left(\mathbb{E}\left\{\exp \left[\theta \int_{0}^{\infty} e^{-\alpha t} \varphi\left(x_{t}, v_{t}\right) \mathrm{d} t\right]\right\}\right)
$$

and the value function is, for $\theta>0$,

$$
u_{\alpha}(\theta, x)=\inf _{v} I_{\alpha}(\theta, x, v)
$$

and we exchange inf with the sup for $\theta<0$. However, in the sequel, we consider only $\theta>0$ for the sake of simplicity.

The aim of this paper is to investigate the asymptotic behavior of $\alpha u_{\alpha}$ when $\alpha$ goes to zero.

Nagai [ா] studied the asymptotic behavior of the finite horizon risk-sensitive control problem, namely,

$$
J(T, x, v)=\frac{1}{\theta} \ln \left(\mathbb{E}\left\{\exp \left[\theta \int_{0}^{T} \varphi\left(x_{t}, v_{t}\right) \mathrm{d} t\right]\right\}\right)
$$


and shows that if $\theta$ is fixed and

$$
u_{T}(t, x)=\inf _{v} J(T-t, x, v)
$$

then

$$
\lim _{T \rightarrow \infty} \frac{1}{T} u_{T}(T, x)=\chi,(\text { constant }),
$$

and

$$
\lim _{T \rightarrow \infty}\left[u_{T}(T, x)-u_{T}(0, x)\right]=z(x), \text { (function), }
$$

where the couple $(\chi, z)$ satisfies the equation

$$
\chi=\Delta z+\theta|D z|^{2}+\inf _{v}\{\varphi+b \cdot \nabla z\} .
$$

Clearly, $(\chi, z)$ may depends on $\theta$.

We will see in Section 2, that the HJB equation for $(\mathbb{\square}: 3)$ is

$$
-\alpha\left(u_{\alpha}+\theta \frac{\partial u_{\alpha}}{\partial \theta}\right)+\Delta u_{\alpha}+\theta\left|\nabla u_{\alpha}\right|^{2}+\inf _{v}\left\{\varphi+b \cdot \nabla u_{\alpha}\right\}=0 .
$$

Comparing (ㅁ.6) and (ㄸ. $)$, we can anticipate that

$$
\alpha\left(u_{\alpha}+\theta \frac{\partial u_{\alpha}}{\partial \theta}\right) \rightarrow \chi(\theta), \text { as } \alpha \rightarrow 0 .
$$

In other words, assume that there exists $\xi(\theta)$ (independent of $x$ ) such that

$$
\alpha u_{\alpha}(\theta, x) \rightarrow \xi(\theta) \text { and } \alpha \frac{\partial u_{\alpha}}{\partial \theta}(\theta, x) \rightarrow \frac{\mathrm{d} \xi(\theta)}{\mathrm{d} \theta},
$$

as $\alpha \rightarrow 0$, we would have, by (ㅁ),

$$
\chi(\theta)=\xi(\theta)+\theta \frac{\mathrm{d} \xi(\theta)}{\mathrm{d} \theta}=\frac{\mathrm{d}}{\mathrm{d} \theta}[\theta \xi(\theta)]
$$

and

$$
\xi(\theta)=\frac{1}{\theta} \int_{0}^{\theta} \chi(r) \mathrm{d} r=\lim _{\alpha} \alpha u_{\alpha}(\theta, x) .
$$

Notice that when $\theta=0$, the equation (ㅍ.]) corresponds to the usual discounted control,

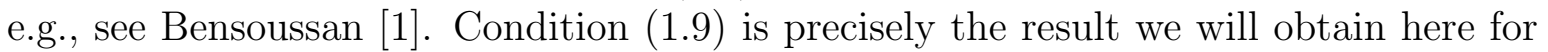
the case of periodic diffusion (or reflected diffusions on a bounded region of $\mathbb{R}^{d}$ ).

The risk-sensitive control problem for diffusion processes (in various cases) has been studied by several authors, particularly in connection with robust control and differential games, for instance, we refer to Jacobson [ [ ], Bensoussan and Van Schuppen [ $⿴ 囗 \mathbf{]}$ ],

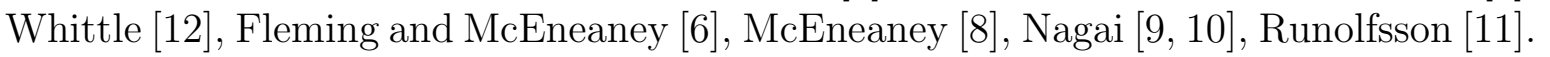

In Section 2, we obtain formally the HJB-equation for (ㅍ.3), and a verification theorem. In Section 3, we study the discounted risk-sensitive problem, and in Section 4 , we consider the asymptotic behavior when the discount factor goes to zero. 


\section{Formal Derivation of the HJB Equation}

We start with

$$
w_{\alpha}(\theta, x)=\inf _{v} \exp \left[\theta I_{\alpha}(\theta, x, v)\right]
$$

Formally, for any $T>0$ and for any Markov control $v_{t}=v\left(x_{t}\right)$, we argue as follows

$$
\begin{aligned}
w_{\alpha}(\theta, x)=\inf _{v} \mathbb{E}_{x}\left\{\operatorname { e x p } \left[\theta \int _ { 0 } ^ { T } e ^ { - \alpha t } \varphi \left(\left(x_{t}, v_{t}\right) \mathrm{d} t+\right.\right.\right. \\
\left.+\theta \int_{T}^{\infty} e^{-\alpha t} \varphi\left(\left(x_{t}, v_{t}\right) \mathrm{d} t\right]\right\}= \\
=\inf _{v} \mathbb{E}_{x}\left\{\operatorname { e x p } \left[\theta \int_{0}^{T} e^{-\alpha t} \varphi\left(\left(x_{t}, v_{t}\right) \mathrm{d} t\right] \times\right.\right. \\
\times \mathbb{E}_{x_{T}}\left\{\exp \left[\theta e^{-\alpha T} \int_{0}^{\infty} e^{-\alpha t} \varphi\left(\left(x_{t}, v_{t}\right) \mathrm{d} t\right]\right\}\right\} .
\end{aligned}
$$

Therefore (formally)

$$
w_{\alpha}(\theta, x)=\inf _{v /[0, T]} \mathbb{E}_{x}\left\{\exp \left[\theta \int_{0}^{T} e^{-\alpha t} \varphi\left(x_{t}, v_{t}\right) \mathrm{d} t\right] w_{\alpha}\left(\theta e^{-\alpha T}, x_{T}\right)\right\}
$$

Using Itô's formula for $w_{\alpha}\left(\theta e^{-\alpha T}, x_{T}\right)$, and taking $T>0$ small, we obtain

$$
-\alpha \theta \frac{\partial w_{\alpha}}{\partial \theta}+\Delta w_{\alpha}+\inf _{v}\left\{\theta \varphi w_{\alpha}+b \cdot \nabla w_{\alpha}\right\}=0,
$$

and clearly $w_{\alpha}(0, x)=1$.

Next, we set $w_{\alpha}=\exp \left(\theta u_{\alpha}\right)$ to deduce

$$
-\alpha\left(u_{\alpha}+\theta \frac{\partial u_{\alpha}}{\partial \theta}\right)+\Delta u_{\alpha}+\theta\left|\nabla u_{\alpha}\right|^{2}+\inf _{v}\left\{\varphi+b \cdot \nabla u_{\alpha}\right\}=0
$$

Remark that one should take

$$
u_{\alpha}(0, x)=\inf _{v} \mathbb{E}_{x}\left\{\int_{0}^{\infty} e^{-\alpha t} \varphi\left(x_{t}, v_{t}\right) \mathrm{d} t\right\}
$$

since, when $\theta$ is small in (ㄹ) we have

$$
I_{\alpha}(\theta, x, v)=\mathbb{E}_{x} \Phi+\theta \mathbb{E}_{x} \Phi^{2}+O\left(\theta^{2}\right)
$$

where

$$
\Phi=\int_{0}^{\infty} e^{-\alpha t} \varphi\left(x_{t}, v_{t}\right) \mathrm{d} t
$$


Theorem 2.1 (implicit assumptions). Let us assume that there exists a smooth function $W(\theta, x)$ such that

$$
-\alpha \theta \frac{\partial W}{\partial \theta}+\Delta W+\inf _{v}\{\theta \varphi W+b \cdot \nabla W\}=0,
$$

and $W(\theta, x) \rightarrow 1$ as $\theta \rightarrow 0$, locally uniform in $x$. Also assume that there exists an optimal control $v^{*}$. Then

$$
W(\theta, x)=w_{\alpha}(\theta, x) .
$$

Proof. To see this, introduce $\theta_{t}$ defined by

$$
\frac{\mathrm{d} \theta_{t}}{\mathrm{~d} t}=-\alpha \theta_{t}, \quad \theta_{0}=\theta
$$

and

$$
\psi_{T}=\exp \left\{\int_{0}^{T} \theta_{t} \varphi\left(x_{t}, v_{t}\right) \mathrm{d} t\right\}
$$

for an arbitrary control $v_{s}$. By means of Feynman-Kac formula we get

$$
\begin{aligned}
& \mathbb{E}_{x}\left\{\psi_{T} W\left(\theta_{T}, x_{T}\right)\right\}=W(\theta, x)+ \\
& \quad+\mathbb{E}_{x}\left\{\int_{0}^{T} \psi_{t}\left[-\alpha \theta \frac{\partial W}{\partial \theta}+\Delta W+\theta \varphi W+b \cdot \nabla W\right] \mathrm{d} t\right\} .
\end{aligned}
$$

From the equation for $W$ the last term is nonnegative, and therefore

$$
W(\theta, x) \leq \mathbb{E}_{x}\left\{W\left(\theta_{T}, x_{T}\right) \exp \left[\theta \int_{0}^{T} e^{-\alpha t} \varphi\left(x_{t}, v_{t}\right) \mathrm{d} t\right]\right\}
$$

Hence, because $\theta_{T} \rightarrow 0$ as $T \rightarrow \infty$ and $W\left(\theta_{T}, x_{T}\right) \rightarrow 1$ (locally uniform in $x_{T}$ ) as $\theta \rightarrow 0$ we deduce

$$
W(\theta, x) \leq \mathbb{E}_{x}\left\{\exp \left[\theta \int_{0}^{\infty} e^{-\alpha t} \varphi\left(x_{t}, v_{t}\right) \mathrm{d} t\right]\right\},
$$

i.e., $W(\theta, x) \leq w_{\alpha}(\theta, x)$.

Similarly, using the optimal control $v^{*}$ we obtain the equality.

Clearly, as a Corollary, using $U$ defined by $W=\exp (\theta U)$ we obtain $U=u_{\alpha}$.

\section{Discounted Risk-sensitive Problem}

Let $(\Omega, \mathcal{F}, P)$ be a probability space with a filtration $\left(\mathcal{F}_{t}: t \geq 0\right)$ and a standard $d$-dimensional $\mathcal{F}_{t}$-Brownian motion process $\left(B_{t}: t \geq 0\right)$. We are given $V$ a compact metric space, $\left.\left.X=\left[\left(\mathbb{R}^{d}\right) \bmod (1)\right] \simeq\right] 0,1\right]^{d}$

$$
b: X \times V \rightarrow \mathbb{R}^{d}, \quad \varphi: X \times V \rightarrow \mathbb{R},
$$


where $b(x, v)$ and $\varphi(x, v)$ are periodic in $x$ with period 1 in each coordinate (as functions defined on $\mathbb{R}^{d}$ ), $b$ is continuous in $X \times V$ and Lipschitz continuous in $x$, namely,

$$
\left|b(x, v)-b\left(x^{\prime}, v\right)\right| \leq M\left|x-x^{\prime}\right|, \quad \forall x, x^{\prime} \in X,
$$

$\varphi$ is continuous and nonnegative.

The state equation is given by

$$
\left\{\begin{array}{l}
\mathrm{d} x_{t}=b\left(x_{t}, v_{t}\right) \mathrm{d} t+\sqrt{2} \mathrm{~d} B_{t}, \quad t>0, \\
x_{0}=x \in X
\end{array}\right.
$$

where $\left(v_{t}: t \geq 0\right)$ is any progressively measurable process with values in $V$.

As above, the cost is given by

$$
I_{\alpha}(\theta, x, v)=\frac{1}{\theta} \ln \mathbb{E}_{x}\left\{\exp \left(\theta \int_{0}^{\infty} \mathrm{e}^{-\alpha t} \varphi\left(x_{t}, v_{t}\right) \mathrm{d} t\right)\right\}
$$

where $\alpha>0$ is the discount factor and $\theta$ is a real parameter. For the sake of simplicity, we will consider only the case $\theta>0$. The optimal cost function is

$$
u_{\alpha}(\theta, x)=\inf _{v} I_{\alpha}(\theta, x, v) .
$$

Remark 3.1. One could avoid the assumption (B2) that $b$ is Lipschitz continuous and then define the state equation using the Girsanov transformation (e.g., see Bensoussan [四, Chapter 6]).

As seen in Section 叉, the HJB-equation for (5.5) is

$$
A_{\theta} u_{\alpha}+\alpha u_{\alpha}=H\left(\theta, x, D u_{\alpha}\right),
$$

with $u_{\alpha}$ periodic in $x$,

$$
\begin{aligned}
& A_{\theta} u:=\alpha \theta \partial_{\theta} u-\Delta u-\theta|D u|^{2}, \\
& H(\theta, x, p):=\inf _{v}\{\varphi(x, v)+b(x, v) \cdot p\},
\end{aligned}
$$

and

$$
u_{\alpha}(0, x)=u_{\alpha}^{0}(x),
$$

with

$$
A_{0} u_{\alpha}^{0}=H\left(0, x, D u_{\alpha}^{0}\right),
$$

and $u_{\alpha}^{0}$ periodic. Note that $D u, \Delta u$ and $\partial_{\theta} u$ denote the gradient in $x$, the Laplacian in $x$, and the partial derivative in $\theta$, respectively.

It is well known (e.g., see Bensoussan and Lions [0, [3]) that (B.8) has a unique solution in $W^{2, p}(X), 2 \leq p<\infty$. Without any lost of generality, we consider (医) with $\theta$ in $] 0,1[$.

First we study an auxiliary equation in $w$, namely,

$$
\alpha \theta \partial_{\theta} w-\Delta w=\inf _{v}\{\theta \varphi w+b \cdot D w\},
$$

with $w$ periodic in $x$ and $w(0, x)=1$. 


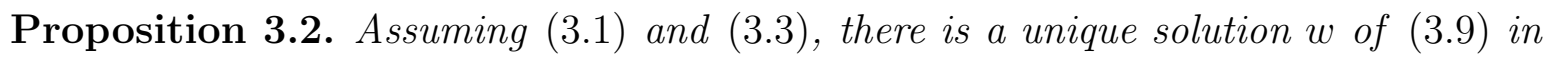
$H^{1}(] 0,1[\times X)$ such that $w$ and $\partial_{\theta} w$ belong to $L^{\infty}(] 0,1[\times X)$.

Proof. We begin with the following equation for $\varepsilon$ in $] 0,1[$,

$$
\begin{aligned}
& \left.\alpha \theta \partial_{\theta} w^{\varepsilon}-\Delta w^{\varepsilon}=\inf _{v}\left\{\theta \varphi w^{\varepsilon}+b \cdot D w^{\varepsilon}\right\}, \quad \theta \in\right] \varepsilon, 1[, \\
& w^{\varepsilon}(\varepsilon, x)=h_{\varepsilon}(x), \quad x \in X
\end{aligned}
$$

with $w^{\varepsilon}$ periodic in $x$ and

$$
h^{\varepsilon}(x)=\mathrm{e}^{\frac{\varepsilon}{\alpha}\|\varphi\|},
$$

where

$$
\|\varphi\|:=\sup _{x, v}|\varphi(x, v)|
$$

and clearly $h_{\varepsilon} \rightarrow 1$ as $\varepsilon \rightarrow 0$.

Since $\theta$ belongs to ] $\varepsilon, 1$ [, equation ( $\mathbf{B} \cdot \mathbf{l})$ can be seen as a standard Cauchy problem and there is a unique solution $w^{\varepsilon}$ in $W_{p}^{1,2}(] \varepsilon, 1[\times X), 2 \leq p<\infty$. Therefore, we can interpret $w^{\varepsilon}(\theta, x)$ as the following optimal cost

$$
w^{\varepsilon}(\theta, x)=\inf _{v} \mathbb{E}_{x}\left\{h_{\varepsilon} \exp \left(\theta \int_{0}^{T_{\varepsilon}} \mathrm{e}^{-\alpha t} \varphi\left(x_{t}, v_{t}\right) \mathrm{d} t\right)\right\}
$$

by applying Itô formula to $\psi_{T} w\left(\theta_{T}, x_{T}\right)$ with

$$
\theta_{t}:=\theta \mathrm{e}^{-\alpha t}, \quad \psi_{T}=\exp \left(\int_{0}^{T} \theta_{t} \varphi\left(x_{t}, v_{t}\right) \mathrm{d} t\right)
$$

and where we have taken

$$
T_{\varepsilon}=\inf \left\{t \geq 0: \theta_{t}=\varepsilon\right\}, \quad \text { i.e. } T_{\varepsilon}=\frac{\ln \left(\frac{\theta}{\varepsilon}\right)}{\alpha} .
$$

Then we deduce

$$
0 \leq w^{\varepsilon}(\theta, x) \leq \mathrm{e}^{\frac{\theta}{\alpha}\|\varphi\|}
$$

for every $\varepsilon>0$.

To show that $\partial_{\theta} w^{\varepsilon}$ is uniformly (in $\varepsilon>0$ ) bounded in $L^{\infty}(] \varepsilon, 1[\times X)$ for a fixed $\alpha>0$, we consider the expression

$$
\begin{aligned}
\mid \mathbb{E}_{x}\left\{h_{\varepsilon} \exp \right. & \left.\left((\theta+\delta) \int_{0}^{T_{\varepsilon}^{\delta}} \mathrm{e}^{-\alpha t} \varphi\left(x_{t}, v_{t}\right) \mathrm{d} t\right)\right\}- \\
& -\mathbb{E}_{x}\left\{h_{\varepsilon} \exp \left(\theta \int_{0}^{T_{\varepsilon}} \mathrm{e}^{-\alpha t} \varphi\left(x_{t}, v_{t}\right) \mathrm{d} t\right)\right\} \mid \leq I_{1}+I_{2},
\end{aligned}
$$


with

$$
(\theta+\delta) \mathrm{e}^{-\alpha T_{\varepsilon}^{\delta}}=\varepsilon, \quad \text { i.e. } \quad T_{\varepsilon}^{\delta}=\frac{\ln \left(\frac{\theta+\delta}{\varepsilon}\right)}{\alpha}
$$

and

$$
\begin{aligned}
& I_{1}=\mid \mathbb{E}_{x}\{\left.h_{\varepsilon} \exp \left((\theta+\delta) \int_{0}^{T_{\varepsilon}^{\delta}} \mathrm{e}^{-\alpha t} \varphi\left(x_{t}, v_{t}\right) \mathrm{d} t\right)\right\}- \\
&-\mathbb{E}_{x}\left\{h_{\varepsilon} \exp \left(\theta \int_{0}^{T_{\varepsilon}^{\delta}} \mathrm{e}^{-\alpha t} \varphi\left(x_{t}, v_{t}\right) \mathrm{d} t\right)\right\} \mid, \\
& I_{2}=\mid \mathbb{E}_{x}\left\{h_{\varepsilon} \exp \left(\theta \int_{0}^{T_{\varepsilon}^{\delta}} \mathrm{e}^{-\alpha t} \varphi\left(x_{t}, v_{t}\right) \mathrm{d} t\right)\right\}- \\
&-\mathbb{E}_{x}\left\{h_{\varepsilon} \exp \left(\theta \int_{0}^{T_{\varepsilon}} \mathrm{e}^{-\alpha t} \varphi\left(x_{t}, v_{t}\right) \mathrm{d} t\right)\right\} \mid,
\end{aligned}
$$

for $\delta>0$ and any arbitrary control. Now

$$
\begin{aligned}
& I_{1} \leq\left|h_{\varepsilon}\right| \mathbb{E}_{x}\left\{\exp \left(\theta \int_{0}^{T_{\varepsilon}^{\delta}} \mathrm{e}^{-\alpha t} \varphi\left(x_{t}, v_{t}\right) \mathrm{d} t\right)\left|\exp \left(\delta \int_{0}^{T_{\varepsilon}^{\delta}} \mathrm{e}^{-\alpha t} \varphi\left(x_{t}, v_{t}\right) \mathrm{d} t\right)-1\right|\right\} \leq \\
& \leq\left|h_{\varepsilon}\right| \delta \frac{\|\varphi\|}{\alpha} \exp \left(\frac{(\theta+\delta)\|\varphi\|}{\alpha}\right)
\end{aligned}
$$

while

$$
\begin{gathered}
I_{2} \leq\left|h_{\varepsilon}\right| \mathbb{E}_{x}\left\{\exp \left(\theta \int_{0}^{T_{\varepsilon}} \mathrm{e}^{-\alpha t} \varphi\left(x_{t}, v_{t}\right) \mathrm{d} t\right)\left|\exp \left(\theta \int_{T_{\varepsilon}}^{T_{\varepsilon}^{\delta}} \mathrm{e}^{-\alpha t} \varphi\left(x_{t}, v_{t}\right) \mathrm{d} t\right)-1\right|\right\} \leq \\
\leq\left|h_{\varepsilon}\right| \exp \left(\frac{\theta\|\varphi\|}{\alpha}\right)\left[\exp \left(\frac{\theta\|\varphi\|}{\alpha}\left(\mathrm{e}^{-\alpha T_{\varepsilon}}-\mathrm{e}^{-\alpha T_{\varepsilon}^{\delta}}\right)\right)-1\right],
\end{gathered}
$$

but $\theta \mathrm{e}^{-\alpha T_{\varepsilon}}=\varepsilon$ so that

$$
\theta \mathrm{e}^{-\alpha T_{\varepsilon}}-\theta \mathrm{e}^{-\alpha T_{\varepsilon}^{\delta}}=\delta \mathrm{e}^{-\alpha T_{\varepsilon}^{\delta}}=\frac{\varepsilon \delta}{\theta+\delta}
$$

and

$$
I_{2} \leq\left|h_{\varepsilon}\right| \exp \left(\frac{\theta\|\varphi\|}{\alpha}\right)\left[\exp \left(\frac{\varepsilon \delta\|\varphi\|}{\alpha(\theta+\delta)}\right)-1\right] .
$$

Similarly for $\delta<0$, and we deduce a bound of the type

$$
\left|w^{\varepsilon}(\theta+\delta, x)-w^{\varepsilon}(\theta, x)\right| \leq C\left|h_{\varepsilon}\right| \mathrm{e}^{\frac{\theta}{\alpha}\|\varphi\|} \frac{\|\varphi\|}{\alpha}|\delta|,
$$

and so $\partial_{\theta} w^{\varepsilon}$ is uniformly (in $\varepsilon>0$ ) bounded for a fixed $\alpha>0$.

Now we show that for any $\theta$ in ] $], 1\left[\right.$ the function $x \mapsto w^{\varepsilon}(\theta, x)$ is bounded in $W^{2, p}(X)$, uniformly with respect to $\varepsilon$ and $\theta$. Indeed, for $\lambda>0$ sufficiently large, we write the equation in $w^{\varepsilon}$ as

$$
-\Delta w^{\varepsilon}+\lambda w^{\varepsilon}=\inf _{v}\left\{\psi^{\varepsilon}(\cdot, v)+b(\cdot, v) \cdot D w^{\varepsilon}\right\}
$$


with $\psi^{\varepsilon}=\theta \varphi w^{\varepsilon}+\lambda w^{\varepsilon}-\alpha \theta \partial_{\theta} w^{\varepsilon}$. Since $w^{\varepsilon}$ and $\partial_{\theta} w^{\varepsilon}$ are bounded uniformly in $\varepsilon$ and $\theta$, classic results show that

$$
\left\|w^{\varepsilon}(\theta, \cdot)\right\|_{W^{2, p}(X)} \leq C
$$

where the constant $C$ depends only on the bounds of $\psi^{\varepsilon}, b$ and the constant $\lambda$.

Define $\tilde{w}^{\varepsilon}$ on $] 0,1[\times X$ as

$$
\tilde{w}^{\varepsilon}(\theta, x)=\left\{\begin{array}{ll}
w^{\varepsilon}(\theta, x), & \theta>\varepsilon, \\
h_{\varepsilon}(x), & \theta \leq \varepsilon,
\end{array},\right.
$$

which satisfies the same estimates (uniformly in $\varepsilon$ ) as $w^{\varepsilon}$, i.e., $\tilde{w}^{\varepsilon} \geq 0$, bounded and continuous in $] 0,1\left[\times X\right.$, with $\partial_{\theta} \tilde{w}^{\varepsilon}$ bounded in $L^{\infty}(] 0,1[\times X)$ and $\tilde{w}^{\varepsilon}(\theta, \cdot)$ bounded in $W^{2, p}(X)$, uniformly in $\theta$. Thus, by extracting a subsequence, we have in particular,

$$
\tilde{w}^{\varepsilon} \rightarrow w \quad \text { in } \quad L^{2}\left(0,1 ; H^{2}(X)\right) \text { weakly, }
$$

and

$$
\partial_{\theta} \tilde{w}^{\varepsilon} \rightarrow \partial_{\theta} w \quad \text { in } \quad L^{2}(] 0,1[\times X) \text { weakly. }
$$

These estimates allow to pass to the limit as $\varepsilon \rightarrow 0$ in

$$
\begin{aligned}
\int_{0}^{1} \alpha \theta\left\langle\partial_{\theta} \tilde{w}^{\varepsilon}, z\right\rangle \mathrm{d} \theta+\int_{0}^{1}\left\langle D \tilde{w}^{\varepsilon}, D z\right\rangle \mathrm{d} \theta- \\
\quad-\int_{0}^{1}\left\langle\inf _{v}\left\{\theta \varphi \tilde{w}^{\varepsilon}+b(\cdot, v) \cdot D \tilde{w}^{\varepsilon}\right\}, z\right\rangle \mathrm{d} \theta=\int_{0}^{\varepsilon}\left\langle\inf _{v}\left\{\theta \varphi h_{\varepsilon}\right\}, z\right\rangle \mathrm{d} \theta
\end{aligned}
$$

to obtain ( $\mathrm{EQ}$. $)$.

We are ready to state

Theorem 3.3. Assume (B.]) (酋), (B.]) such that $u$ and $\partial_{\theta} u$ belong to $L^{\infty}(] 0,1[\times X)$, the functions $x \mapsto u(\theta, x)$ belong to $W^{2, p}(X)$ and $u=u_{\alpha}(\theta, x)$ given by (咠).

Proof. By means of the Itô formula, first with an arbitrary control and next with $\hat{v}$ defined as the minimizer

$$
\hat{v}=\operatorname{argmin}\{\theta \varphi(\cdot, v) w+b(\cdot, v) \cdot D w\},
$$

we obtain

$$
w_{\alpha}(\theta, x)=\inf _{v} \mathbb{E}_{x}\left\{\exp \left(\theta \int_{0}^{\infty} \mathrm{e}^{-\alpha t} \varphi\left(x_{t}, v_{t}\right) \mathrm{d} t\right)\right\} .
$$

Now define $u$ as

$$
\mathrm{e}^{\theta u}=w_{\alpha}, \quad \theta>0
$$


to get

$$
\alpha\left(u+\theta \partial_{\theta} u\right)-\Delta u-\theta|D u|^{2}=\inf _{v}\{\varphi(\cdot, v)+b(\cdot, v) \cdot D u\} .
$$

For $\theta=0$, we define $u(0, x)=\bar{u}$ as the solution of

$$
\alpha \bar{u}-\Delta \bar{u}=\inf _{v}\{\varphi(\cdot, v)+b(\cdot, v) \cdot D \bar{u}\}, \quad \bar{u} \in W^{2, p},
$$

which is known to exist (see Bensoussan and Lions [[]]).

From the definition of $u$ we obtain

$$
u(\theta, x)=\inf _{v} I_{\alpha}(\theta, x, v),
$$

which conclude the proof, in view of the regularity of $w_{\alpha}$.

\section{Asymptotics}

The first step is to obtain estimates on $u_{\alpha}$ independent of $\alpha$.

-Estimate of $\alpha u_{\alpha}$ :

As seen before, for $\theta>0$ and $\varphi \geq 0$, we have

$$
1 \leq w_{\alpha} \leq \mathrm{e}^{\frac{\theta\|\varphi\|}{\alpha}}
$$

and therefore

$$
0 \leq u_{\alpha} \leq \frac{\|\varphi\|}{\alpha}
$$

So

$$
0 \leq \alpha u_{\alpha}(x) \leq\|\varphi\|, \quad \forall \alpha>0
$$

-Estimate of $\alpha\left(u_{\alpha}+\theta \partial_{\theta} u_{\alpha}\right)=\alpha \partial_{\theta}\left(\theta u_{\alpha}\right)$ :

Define

$$
\begin{aligned}
\Phi_{\alpha} & :=\int_{0}^{\infty} \mathrm{e}^{-\alpha t} \varphi\left(x_{t}, v_{t}\right) \mathrm{d} t \\
\Psi_{\alpha} & :=\ln \mathbb{E}_{x}\left\{\mathrm{e}^{\theta \Phi_{\alpha}}\right\}=\ln \int_{\Omega} \mathrm{e}^{\theta \Phi_{\alpha}(\omega)} P_{x}(\mathrm{~d} \omega) .
\end{aligned}
$$

Clearly

$$
\Psi_{\alpha}(x, v, \theta+\delta)=\Psi_{\alpha}(x, v, \theta)+\delta \partial_{\theta} \Psi_{\alpha}(x, v, \theta+\eta \delta),
$$

for some $\eta$ in $(0,1)$. Since

$$
\partial_{\theta} \Psi_{\alpha}=\frac{\mathbb{E}_{x}\left\{\Phi_{\alpha} \mathrm{e}^{\theta \Phi_{\alpha}}\right\}}{\mathbb{E}_{x}\left\{\mathrm{e}^{\theta \Phi_{\alpha}}\right\}},
$$


if $K=\|\varphi\|$ then we have

$$
0 \leq \partial_{\theta} \Psi_{\alpha} \leq \frac{K \mathbb{E}_{x}\left\{\mathrm{e}^{\theta \Phi}\right\}}{\alpha \mathbb{E}_{x}\left\{\mathrm{e}^{\theta \Phi}\right\}}=\frac{K}{\alpha},
$$

and

$$
\left|\Psi_{\alpha}(x, v, \theta+\delta)-\Psi_{\alpha}(x, v, \theta)\right| \leq|\delta| \frac{K}{\alpha} .
$$

Therefore

$$
\left|(\theta+\delta) u_{\alpha}(\theta+\delta, x)-\theta u_{\alpha}(\theta, x)\right| \leq|\delta| \frac{\theta K}{\alpha}
$$

So

$$
\left|\partial_{\theta}\left(\theta u_{\alpha}(\theta, x)\right)\right| \leq \frac{\theta K}{\alpha},
$$

i.e., $\alpha \partial_{\theta}\left(\theta u_{\alpha}\right)$ is bounded uniformly in $\alpha$.

-Estimate of $\left|D u_{\alpha}\right|_{L^{2}}$ :

The equation in $u_{\alpha}$ can be written as

$$
-\Delta u_{\alpha}-b_{\alpha} \cdot D u_{\alpha}=\theta\left|D u_{\alpha}\right|^{2}+\psi_{\alpha}-\alpha u_{\alpha},
$$

with

$$
\begin{gathered}
b_{\alpha}=b\left(x, v_{\alpha}\right), \quad \psi_{\alpha}=\varphi\left(x, v_{\alpha}\right)-\alpha \theta \partial_{\theta} u_{\alpha}, \\
v_{\alpha}(x)=\operatorname{argmin}\left\{\varphi(\cdot, v)+b(\cdot, v) \cdot D u_{\alpha}(x)\right\} .
\end{gathered}
$$

Let $m_{\alpha}$ be the density invariant probability measure corresponding to the operator $-\Delta-b_{\alpha} \cdot D$ (e.g., see Bensoussan [प] ), which satisfies

$$
0<\delta_{0} \leq m_{\alpha} \leq \delta_{1}
$$

Multiplying (4.3) by $m_{\alpha}$ and using the equation for $m_{\alpha}$, we deduce

$$
0=\theta \int_{X}\left|D u_{\alpha}\right|^{2} m_{\alpha} \mathrm{d} x+\int_{X}\left(\psi_{\alpha}-\alpha u_{\alpha}\right) m_{\alpha} \mathrm{d} x .
$$

Since $\delta_{0}$ and $\delta_{1}$ depend only on the $L^{\infty}$ norm of $b$, they are independent of $\alpha$ and $\theta$. Therefore (4.4) gives

$$
\theta\left|D u_{\alpha}\right|_{L^{2}(X)}^{2} \leq C, \quad \forall \alpha, \theta,
$$

i.e., a bound on $\left|D u_{\alpha}\right|_{L^{2}(X)}$ uniformly in $\alpha>0$ and $\theta$ in $[\varepsilon, 1]$, for every $\varepsilon>0$.

\section{-Estimate of $u_{\alpha}-\bar{u}_{\alpha}$ :}

Let us define

$$
\bar{u}_{\alpha}(\theta):=\int_{X} u_{\alpha}(\theta, x) \mathrm{d} x \quad \text { and } \quad \Lambda_{\alpha}(\theta, x):=u_{\alpha}(\theta, x)-\bar{u}_{\alpha}(\theta) .
$$


The equation for $\Lambda_{\alpha}$ is

$$
-\Delta \Lambda_{\alpha}=-\alpha \partial_{\theta}\left(\theta u_{\alpha}\right)+\theta\left|D \Lambda_{\alpha}\right|^{2}+\inf _{v}\left\{\varphi(\cdot, v)+b(\cdot, v) \cdot D \Lambda_{\alpha}\right\} .
$$

and by Poincaré inequality we have

$$
\left|\Lambda_{\alpha}\right|_{L^{2}(X)} \leq C\left|D u_{\alpha}\right|_{L^{2}(X)} .
$$

Considering $\theta$ as a parameter in (4.6) and since $\alpha \partial_{\theta}\left(\alpha u_{\alpha}\right)$ is bounded, we have

$$
\sqrt{\theta}\left|\Lambda_{\alpha}\right|_{L^{2}(X)} \leq C
$$

moreover, we can mimic the arguments in Lemmas 4.7 and 4.8 of Bensoussan and Frehse [5] to obtain

$$
\sqrt{\theta}\left|\Lambda_{\alpha}\right|_{L^{\infty}(X)} \leq C
$$

for some constant $C>0$, uniformly in $\alpha$ and $\theta$. Furthermore, considering $z_{\alpha}(\theta, x)=$ $\theta \Lambda_{\alpha}(\theta, a)$, which satisfies

$$
-\Delta z_{\alpha}=-\alpha \theta \partial_{\theta}\left(\theta u_{\alpha}\right)+\left|D z_{\alpha}\right|^{2}+\inf _{v}\left\{\theta \varphi(\cdot, v)+b(\cdot, v) \cdot D z_{\alpha}(\theta, \cdot)\right\},
$$

so that one can apply Theorem 3.7 of Bensoussan and Frehse [5] to deduce

$$
\left\|z_{\alpha}\right\|_{C^{\delta}(X)} \leq C
$$

i.e.,

$$
\theta\left|\Lambda_{\alpha}\right|_{C^{\delta}(X)} \leq C
$$

for some constant $C>0$, uniformly in $\alpha$ and $\theta$.

-Passage to the limit a $\alpha \rightarrow 0$ :

(a) First we look at $\alpha u_{\alpha}(\theta, x)$. In view of (4.]), (4.2) and (4.8), taking a sub-sequence we have

$$
\alpha u_{\alpha} \rightarrow \xi
$$

uniformly on every compact subset of $Q=] 0,1[\times X$. Let us show that $\xi$ does not depend on $x$. Indeed, since

$$
\sqrt{\theta} \Lambda_{\alpha}=\sqrt{\theta}\left[u_{\alpha}(\theta, x)-\bar{u}_{\alpha}(\theta)\right]
$$

is bounded, we have $\alpha \sqrt{\theta} \Lambda_{\alpha} \rightarrow 0$ and therefore

$$
\lim _{\alpha \rightarrow 0} \alpha\left[u_{\alpha}(\theta, x)-\bar{u}_{\alpha}(\theta)\right]=0, \quad \forall x \in X, \theta>0 .
$$

On the other hand, since $u_{\alpha}(0, x)=u_{\alpha}^{0}(x)$ we know that $\alpha u_{\alpha}^{0}(x)$ must converge to a constant too. 
Now, since $\theta \partial_{\theta}\left(\alpha u_{\alpha}\right)$ is bounded, we deduce that

$$
\theta \partial_{\theta}\left(\alpha u_{\alpha}\right) \rightarrow \theta \frac{d \xi}{d \theta}
$$

weakly-star in $L^{\infty}$,

(b) Then we pass to the limit in the equation of $\Lambda_{\alpha}$, for each $\theta>0$ fixed. By means of the equation (4.6) and the previous bounds on $u_{\alpha}$, in particular (4.2), (4.5) and (4.8), we can find a subsequence such that

$$
\Lambda_{\alpha} \rightarrow u \text { in } H^{1}(X) \text { weakly and } L^{\infty}(X) \text { strongly }
$$

as $\alpha \rightarrow 0$. Therefore

$$
\int_{X} \Delta \Lambda_{\alpha}\left(\Lambda_{\alpha}-u\right) \mathrm{d} x \rightarrow 0
$$

since $\Delta \Lambda_{\alpha}$ is bounded in $L^{1}(X)$. This is,

$$
\int_{X} D \Lambda_{\alpha} \cdot D \Lambda_{\alpha} \mathrm{d} x \rightarrow \int_{X} D \Lambda_{\alpha} \cdot D u \mathrm{~d} x
$$

However, due to the weak convergence in $H^{1}(X)$ we have

$$
\int_{X} D \Lambda_{\alpha} \cdot D u \mathrm{~d} x \rightarrow \int_{X} D u \cdot D u \mathrm{~d} x
$$

which yields

$$
\int_{X}\left|D \Lambda_{\alpha}-D u\right|^{2} \mathrm{~d} x \rightarrow 0
$$

i.e., $\Lambda_{\alpha} \rightarrow u(\theta, \cdot)$ strongly in $H^{1}(X)$.

Hence, if we call $\chi(\theta)$ the limit of $\alpha \partial_{\theta}\left(\theta u_{\alpha}\right)$ we see that the couple $(\chi, u)$ satisfies

$$
\left\{\begin{array}{l}
\chi-\Delta u=\theta|D u|^{2}+\inf _{v}\{\varphi(\cdot, v)+b(\cdot, v) \cdot D u(\cdot)\}, \quad u \in H^{1}(X), \\
\int_{X} u(\theta, x) \mathrm{d} x=0, \quad \forall \theta>0 .
\end{array}\right.
$$

But form Nagai [ா]] (who treats a more difficult case in $\mathbb{R}^{d}$ and unbounded $\varphi$, and therefore the result applies a fortiori to our simple case) there exists a unique pair $(\chi, u)$ satisfying (4.]) and

$$
\chi(\theta)=\lim _{T \rightarrow \infty} \frac{u(T, x)}{T}
$$

with $u(T, x)$ given by $(\mathbb{\square} . \mathbf{b})$. Therefore we conclude that

$$
\frac{\mathrm{d}(\theta \xi(\theta))}{\mathrm{d} \theta}=\chi(\theta),
$$


which gives

$$
\xi(\theta)=\frac{1}{\theta} \int_{0}^{\theta} \chi(r) \mathrm{d} r
$$

i.e.,

$$
\lim _{\alpha \rightarrow 0} \alpha u_{\alpha}(\theta, x)=\frac{1}{\theta} \int_{0}^{\theta} \chi(r) \mathrm{d} r
$$

We have shown the desired result summarized as

Theorem 4.1. Under the assumptions of Section 国 we have

$$
\begin{aligned}
& \lim _{\alpha \rightarrow 0} \alpha \partial_{\theta}\left(\alpha u_{\alpha}(\theta, x)\right)=\chi(\theta), \\
& \lim _{\alpha \rightarrow 0}\left[u_{\alpha}(\theta, x)-\int_{X} u_{\alpha}(\cdot, x) \mathrm{d} x\right]=u(\theta, x),
\end{aligned}
$$

where $(\chi, u)$ is the unique solution of (4.T),

$$
\chi(\theta)=\liminf _{T \rightarrow \infty} \frac{1}{T} \inf _{v}\left[\frac{1}{\theta} \ln \mathbb{E}_{x}\left\{\exp \left(\theta \int_{0}^{T} \varphi\left(x_{t}, v_{t}\right) \mathrm{d} t\right)\right\}\right]
$$

and (4.T]) holds.

To conclude, let us mention that certainly, the above result remain true for reflected diffusion processes in a bounded region of $\mathbb{R}^{d}$. The case in the whole space $\mathbb{R}^{d}$ or diffusion with jumps requires a more elaborated technique, and it may be the subject of future research.

\section{References}

[1] A. Bensoussan, Perturbation methods in optimal control, Wiley, New York, 1988.

[2] A. Bensoussan and J.L. Lions, Applications des inéquations variationnelles en contrôle stochastique, Dunod, Paris 1978.

[3] A. Bensoussan and J.L. Lions, Contrôle impulsionnel et inéquations quasi variationnelles, Dunod, Paris 1982.

[4] A. Bensoussan and J.H. Van Schuppen, Optimal control of partially observable stochastic systems with an exponential of integral performance index, SIAM J. Control Optim., 23 (1985), 599-613.

[5] A. Bensoussan and J. Frehse, Regularity results for nonlinear elliptic systems and applications, Springer-Verlag, New-York, 2002.

[6] W.H. Fleming and W.M. McEneaney, Risk-sensitive control on an infinite time horizon, SIAM J. Control Optim., 33 (1995), 1881-1915. 
[7] D.H. Jacobson, Optimal stochastic linear systems with exponential performance criteria and relation to detewninistic differential games, IEEE Trans. Automat. Control, AC-18 (1973), 124-131.

[8] W.M. McEneaney, Connections between risk-sensitive stochastic control, differential games and $H^{\infty}$-control: the non linear case, Brown University, PhD Thesis, 1993.

[9] H. Nagai, Ergodic control problems on the whole Euclidean space and convergence of symmetric diffusions, Forum Math., 4 (1992), 159-173.

[10] H. Nagai, Bellman equations of risk-sensitive control, SIAM J. Control Optim. 34 (1996), 74-101.

[11] T. Runolfsson, Stationary risk-sensitive LQG control and its relation to LQG and H-infinity control, Proc. 29th CDC Conference, Honolulu, HI, 1990, 1018-1023.

[12] P. Whittle, Risk-sensitive Optimal Control, Wiley, New-York, 1990. 